\title{
On the Achievable Degrees of Freedom of Partially Cooperative X Networks with Delayed CSIT
}

\author{
Zhao Wang, Chao Wang, Ming Xiao, and Mikael Skoglund \\ Communication Theory Lab. Royal Institute of Technology (KTH) \\ Email: \{zhaowang, chaowang, mingx, skoglund\}@kth.se, Stockholm, 10044, Sweden
}

\begin{abstract}
We investigate the achievable degrees of freedom (DoF) in $K$-user $\mathbf{X}$ networks ( $K \times K \mathbf{X}$ networks) with delayed channel state information at transmitters (CSIT), where partial cooperation (i.e. message sharing) is potentially allowed among transmitters. We consider two possible cooperation scenarios. In the first scenario one of the transmitters serves as a super node which can obtain the messages of the other transmitters. By proper interference alignment (IA) design, we prove that a DoF $\frac{2 K}{K+1}$ can be achieved almost surely. In the second scenario, there is no super node but each transmitter shares its message to its left-side neighbor. We show that when $K=3$, DoF $\frac{7}{5}$ is achievable. In both cases, the achieved DoF are shown to be improved compared with non-cooperative X networks. Moreover, we use a simple example to show that sharing a subset of messages may also improve DoF.
\end{abstract}

\section{INTRODUCTION}

In multi-user wireless communications, the knowledge of channel state information at transmitters (CSIT) is important for the multiplexing gain (or degrees of freedom). For example, consider a $K$-user $(K \times K \mathrm{X}$ networks $) \mathrm{X}$ network with $K$ single-antenna transmitters and $K$ single-antenna receivers, where each transmitter intends to send an independent message to each receiver [1]. Without CSIT, the achievable sum degree of freedom (DoF) is only one. Thus, orthogonal transmission schemes such as time-division-multiple-access (TDMA) attains the optimal performance in terms of DoF. However, with global channel state information (CSI), an elegant interference alignment scheme can achieve $\operatorname{DoF} \frac{K^{2}}{2 K-1}$ in such a network [1]. The result reveals the importance of CSIT. Besides CSIT, cooperation among multiple transmitters is also important for DoF improvement. For instance, consider sharing codebook among all $K$ transmitters in the $\mathrm{X}$ network, the $K$-user $\mathrm{X}$ network is transferred into a $K$-user MIMO broadcast channel, in which the optimal sum DoF increases from $\frac{K^{2}}{2 K-1}$ to $K$ with CSIT. Observing the gaps among these results, the importance of CSIT and cooperation should be both emphasized.

In practice, CSI is obtained by training sequence estimation at receivers while CSIT is typically obtained via feedback links from receivers to transmitters. Quantized CSI is commonly used in the feedback designs, and references [2] [3] [4] show the optimal DoF can be guaranteed with high quantization resolution. Recently, there is interest in the scenarios where channel coherence time is far shorter than the feedback delay such that CSI is completely outdated when obtained by transmitters. Maddah-Ali and Tse proposed a delayed CSIT model in [5] to discuss this scenario. In [5], for the $K$ - user MIMO broadcast channel which has a $K$-antenna transmitter and $K$ single-antenna receivers, DoF $\frac{K}{1+\frac{1}{2}+\frac{1}{3}+\cdots+\frac{1}{K}}$ can be achieved with delayed CSIT. However, the antenna cooperation at transmitter will not always be available due to practical limitations. This motivates the research of distributed processing at transmitters. By distributing $K$ antennas at $K$ distinct transmitters, we study the $K$-user $\mathrm{X}$ networks in this paper. In [6] [7], the $K$-user X networks are shown to achieve $\frac{4}{3}-\frac{2}{3(3 K-1)}$ DoF with delayed CSIT (the optimal DoF is still unknown). This performance gap leads to the intention of seeking user cooperation. Nevertheless, cooperation among all transmitters demands a high system complexity. Thus, cooperation among certain transmitters (partial cooperation) has practical motivation. In [8], message sharing is discussed as a potential scenario of partial cooperation in the 2 -user MIMO X channel with global CSI. It is natural to raise the question if message sharing can improve the sum DoF of $\mathrm{X}$ networks with delayed CSIT. We discuss this problem here.

We first consider the scenario that one super-node transmitter exists in the $K$-user $\mathrm{X}$ networks where the message sets of the other transmitters can be accessed by the super-node transmitter and there is no cooperation among other nodes. We call these networks super-node partially cooperative $K$ user X networks. We show that in such networks proper IA schemes can achieve $\frac{2 K}{K+1}$ DoF with delayed CSIT. Second, we consider the scenario where no super-node exists but each transmitter can share its message to its left-side neighbor. That is, transmitter $i$ knows the message of transmitter $i+1$. We refer this network neighbor-node partially cooperative $\mathrm{X}$ network. In this scenario, for 3-user $\mathrm{X}$ network, we show that DoF $\frac{7}{5}$ can be achieved by IA. Both schemes lead to DoF improvement comparing to the networks without transmitter cooperation. Moreover, considering the fact that message sharing is normally provided by capacity-limited backhauls among transmitters, we study the scenario that full message sharing may not be available. One example is given to show subset messages sharing is also sufficient to improve the DoF.

\section{System Model}

We consider $K$-user $\mathrm{X}$ networks, where each of $K$-single antenna transmitters intends to send independent messages to all $K$ single-antenna receivers. There are $K^{2}$ independent messages totally. $W_{k i}(i, k=1,2, \ldots, K)$ represents a message from transmitter $i$ to receiver $k$. Let $R_{k i}(P)$ represent the rate of $W_{k i}$ subject to the power constraint $P$, and $n$ represent the 


$$
\mathcal{D}^{d-C S I}=\left\{\left[d_{k i}\right] \in \mathbf{R}_{+}^{K^{2}}: \forall\left[\omega_{k i}\right] \in \mathbf{R}_{+}^{K^{2}} \sum_{i, k \in\{1,2, \ldots, K\}} \omega_{k i} d_{k i} \leq \limsup _{P \rightarrow \infty}\left[\sup _{\left[R_{k i}(P)\right]} \sum_{i, k \in\{1,2, \ldots, K\}}\left[\omega_{k i} R_{k i}(P)\right] \frac{1}{\log (P)}\right]\right\}
$$

transmitting block length. $2^{n R_{k i}(P)}$ is the cardinality of the message set of $W_{k i}$. A rate matrix $\left[R_{k i}(P)\right]$ is achievable if the average probability of error for all messages can be made arbitrarily small when $n \rightarrow \infty$. We define the DoF region of the $K$-user $\mathrm{X}$ networks $\mathcal{D}^{d-C S I}[1]$ at the top line of this page. Here we study the sum DoF of the considered $X$ network, which can be defined as:

$$
\eta_{X}^{d-C S I}(K)=\max _{\mathcal{D}^{d-C S I}} \sum_{i, k \in\{1,2, \ldots, K\}} d_{k i} .
$$

The input and output relation at time slot $t$ is

$$
y^{[k]}(t)=\sum_{i=1}^{K} h^{k i}(t) x_{i}(t)+n^{[k]}(t), \quad k=1,2, \ldots, K,
$$

where $y^{[k]}(t)$ is the received signal at receiver $k, n^{[k]}(t) \sim$ $\mathcal{C N}\left(0, N_{0}\right)$ is white Gaussian noise, and $h^{k i}(t) \in \mathbf{C}$ is the independent and identically distributed (i.i.d) Rayleigh fading channel coefficient from transmitter $i$ to receiver $k . x_{i}(t)$ is the signal sent by transmitter $i$. Furthermore, we assume receiver $k$ has perfect and instantaneous knowledge of its directly connected channel $h^{k i}(t)(\forall i=1,2, \ldots, K)$. Then, receivers feed the perfect CSI back to transmitters via one time slot delay broadcasting. We also assume that the receivers can obtain the channel state of the other receivers through the broadcasting after some delay. Consequently, at time slot $t+1, h^{k i}(t)(i, k=1,2, \ldots, K)$ are available at all nodes in the network. In what follows, we define the two cooperation scenarios.

1) Super-node Partial Cooperation: In the $K$-user $\mathrm{X}$ networks, we define $M(i)(i=1,2, \ldots, K)$ as the message set of transmitter $i$, and $\tilde{M}(i) \subseteq M(i)$ denotes the subset of $M(i)$. Moreover, there is one super-node among the $K$ transmitters that can access the message sets of other $K-1$ transmitters. Without the loss of generality, we assume that transmitter 1 is the super-node. Message sets $\tilde{M}(2), \tilde{M}(3), \ldots, \tilde{M}(K)$ can be accessed by transmitter- 1 due to message sharing. We call the networks with above properties as super-node partially cooperative $\mathrm{X}$ network. Fig. 1 illustrates a 3 -user example. A possible scenario for this 3 -user case could be as follows: a single-antenna transmitter intends to send independent messages to three single-antenna receivers by the help of two relay nodes. Capacity-limited backhauls connect the transmitter and relays to provide some messages from transmitter to relays. Thus, the transmitter knows all messages in the network to be a super node while each relay knows different message set. We shall study the achievable DoF of this network in Section III with delayed CSIT.

2) Neighbor-node Partial Cooperation: We consider the scenarios where each transmitter can share its message to its

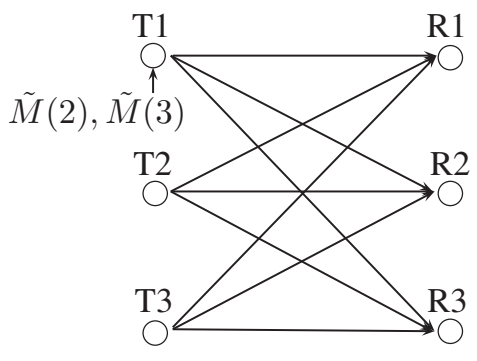

Fig. 1. Three user super-node partially cooperative $\mathrm{X}$ network with delayed CSIT (feedback links are omitted for concision).

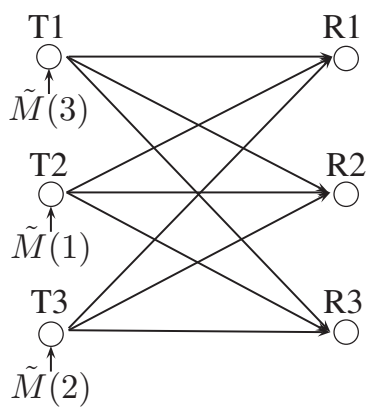

Fig. 2. Three user neighbor-node partially cooperative $\mathrm{X}$ network with delayed CSIT (feedback links are omitted for concision).

left-side neighbor. Specifically, transmitter $i$ knows the messages from transmitter $i+1$. For instance, the network when $K=3$ is shown in Fig. 2. One example of the considered system is the $K$ transmitters form a circle geographically. Capacity-limited backhaul connects transmitters $i+1$ and $i(\forall i=1, \ldots, K-1)$ so that transmitter $i+1$ can share its message set $\tilde{M}(i+1)$ to transmitter $i$ through it (the same situation also applies to the transmitter 1 and its left neighbor transmitter $K$ ). The achievable DoF of this network will be studied in Section IV.

\section{Super-node Partially Cooperative X Networks With Delayed CSIT}

In this section, we study the achievable sum DoF of the super-node partial cooperation scenario. We first give the following results:

Theorem 1. The DoF of $K$-user super-node partially cooperative $\mathrm{X}$ network with delayed CSIT is $\eta_{X}^{d-C S I}(K) \geq \frac{2 K}{K+1}$ almost surely, if $\tilde{M}(i)=M(i), \forall i=2,3, \ldots, K$.

Proof: We present our achievable scheme with respect to $K$.

- For $K=2$, the achievable scheme is the scheme in [5] to obtain the optimal DoF $\frac{4}{3}$ almost surely. We omitted the details for simplicity. 
- For $K=3$, we use the following scheme to achieve DoF $\frac{2 \times 3}{3+1}=\frac{3}{2}$. We divide the transmission into two phases, in which independent messages from all transmitters are sent at phase one and the super-node delivers desired equations (linear combination of transmitted signals) at phase two.

Phase One: Three time slots are used in the first phase. At time slot $k(k=1,2,3)$, independent messages $\mu_{k}, \nu_{k}, \gamma_{k}$ intending for receiver $k$ are transmitted from transmitter 1,2 and 3, respectively. After three time slots, we define the message sets are $M(2)=\left\{\nu_{1}, \nu_{2}, \nu_{3}\right\}$ and $M(3)=\left\{\gamma_{1}, \gamma_{2}, \gamma_{3}\right\}$. Moreover, the received signals at each receiver are:

Receiver 1: The received signals at receiver-1 are

$$
\begin{gathered}
y^{[1]}(k)=h_{11}(k) \mu_{k}+h_{12}(k) \nu_{k}+h_{13}(k) \gamma_{k}+n^{[1]}(k) ; \\
k=1,2,3 .
\end{gathered}
$$

We focus on the high signal to noise ratio (SNR) regime such that thermal noise is ignored in $y^{[1]}(k)$ for simplicity. We further represent the received signal by linear equations, i.e., $y^{[1]}(1)$ is represented by $L_{1}\left(\mu_{1}, \nu_{1}, \gamma_{1}\right) ; y^{[1]}(2)$ is represented by $L_{4}\left(\mu_{2}, \nu_{2}, \gamma_{2}\right)$ and $y^{[1]}(3)$ is represented by $L_{7}\left(\mu_{3}, \nu_{3}, \gamma_{3}\right)$, where the linear equations are:

$$
\begin{aligned}
& L_{1}\left(\mu_{1}, \nu_{1}, \gamma_{1}\right)=h_{11}(1) \mu_{1}+h_{12}(1) \nu_{1}+h_{13}(1) \gamma_{1} ; \\
& L_{4}\left(\mu_{2}, \nu_{2}, \gamma_{2}\right)=h_{11}(2) \mu_{2}+h_{12}(2) \nu_{2}+h_{13}(2) \gamma_{2} ; \\
& L_{7}\left(\mu_{3}, \nu_{3}, \gamma_{3}\right)=h_{11}(3) \mu_{3}+h_{12}(3) \nu_{3}+h_{13}(3) \gamma_{3} .
\end{aligned}
$$

Receiver 2: By the similar notations, the received signals of time slot $1,2,3$ at receiver 2 can be represented as $L_{2}\left(\mu_{1}, \nu_{1}, \gamma_{1}\right), L_{4}\left(\mu_{2}, \nu_{2}, \gamma_{2}\right)$ and $L_{8}\left(\mu_{3}, \nu_{3}, \gamma_{3}\right)$, respectively.

Receiver 3: Similarly, we use $L_{3}\left(\mu_{1}, \nu_{1}, \gamma_{1}\right), L_{6}\left(\mu_{2}, \nu_{2}, \gamma_{2}\right)$ and $L_{9}\left(\mu_{3}, \nu_{3}, \gamma_{3}\right)$ to represent the received linear equations of time slot 1,2 and 3 at receiver 3 , respectively.

Now we observe the linear equations at each receiver. For receiver-1, obtaining $L_{1}$ (we use $L_{i}$ as an abbreviation for $L_{i}(\mu, \nu, \gamma)$ ) is not enough for solving the three variables $\mu_{1}, \nu_{1}, \gamma_{1}$. Two more equations are desired for decoding, i.e., $L_{2}$ and $L_{3}$. Similarly, $L_{4}, L_{6}$ are desired by receiver 2 , and $L_{7}$, $L_{8}$ are desired by receiver 3 for decoding. Thus the problem transfers into how to deliver these equations in the next phase. In order to recover these sent equations, delayed CSIT is fed back to each transmitter to provide the channel coefficients that appear in these equations. We note that it is not sufficient to recover the desired equations at all transmitters due to the lack of the knowledge of the messages from other transmitters. However, since message sets $M(2), M(3)$ are available at the super-node, it is possible for transmitter 1 to recover all desired equations. Based on these observations, we have phase two.

Phase Two: Transmitter 1 sends information at phase two while the other transmitters remain silence. In order to use the overheard equations at receivers efficiently, the desired 6 equations are coupled into three linear combinations, i.e., $L_{2}+L_{4}, L_{3}+L_{7}$ and $L_{6}+L_{8}$. At time slot $4, L_{2}+L_{4}$ are sent by transmitter 1 . By eliminating the overheard equation $L_{4}$, the desired equation $L_{2}$ appears at receiver 1 . At the same time, the desired equation $L_{4}$ appears at receiver 2 by eliminating the overheard equation $L_{2}$. Two independent linear equations are delivered within one time slot. Following the same approach, $L_{3}+L_{7}, L_{6}+L_{8}$ are delivered from the super-node at time slots 5 and 6 , respectively. Consequently, after time slot 6 each receiver has collected three independent equations for decoding its desired three variables. The achieved DoF is $\frac{9}{6}=\frac{3}{2}$ for 3 -user X network.

- The generalization from 3-user case to $K$-user $(K>3)$ case follows the same routine as $K=3$. We briefly discuss it here. Phase one takes $K$ time slots, within which $K^{2}$ independent messages are sent following the same signal pattern as 3-user case. After phase one, each receiver needs $K-1$ independent equations for successful decoding its $K$ desired variables so that a total $K(K-1)$ equations are desired in phase two. By a similar combining, at each time slot of phase two one linear combination of two independent equations is delivered by the super-node. Thus, $\frac{K(K-1)}{2}$ time slots are needed for Phase Two. The achieved DoF is $\frac{K^{2}}{K+\frac{K(K-1)}{2}}=\frac{2 K}{K+1}$.

By super-node partial cooperation, our achieved DoF $\frac{2 K}{K+1}>\frac{4}{3}-\frac{2}{3(3 K-1)}$, where the right hand side is achieved in non-cooperative $K$-user $\mathrm{X}$ networks with delayed CSIT [6]. Specifically, $\frac{3}{2}$ and $\frac{8}{5}$ DoFs can be achieved for $K=3$ and $K=4$, respectively, in our scheme. As comparison, in [6], $\frac{5}{4}$ and $\frac{14}{11}$ DoF are achieved in corresponding networks. Based on these results, DoF improvement is obtained by partial cooperation.

\section{Neighbor-node Partially Cooperative X NETWORK WITH DELAYED CSIT}

In this section, we consider the $\mathrm{X}$ network where the supernode is unavailable. We first consider a neighbor-node partial cooperation scheme and then we study the scenario that only partial message sets can be shared through cooperation.

\section{A. 3-user X Network with Partial Cooperation among Neigh- bors}

As illustrated in Fig. 2, a neighbor message sharing scheme is proposed in 3 -user X network where $\tilde{M}(1), \tilde{M}(2)$ and $\tilde{M}(3)$ are provided at transmitter 2, 3 and 1, respectively (we call this left-side neighbor message sharing). Specifically, we consider full message set sharing in this part, i.e., $\tilde{M}(i)=M(i)$ for $i=1,2,3$. We give the following theorem:

Theorem 2. The DoF of 3-user neighbor-node partially cooperative X networks with delayed CSIT is $\eta_{X}^{d-C S I}(3) \geq \frac{7}{5}$ almost surely, when $\tilde{M}(i)=M(i), \forall i=1,2,3$.

Proof: We present the achievable scheme as follows. The transmission can be designed into two phases. Independent messages are transmitted in phase one and desired equations are delivered in phase two.

Phase One: 9 time slots are used in this phase, and every three time slots are allocated as one round to transmit the intended messages of one receiver from all transmitters. The three-round transmission is designed as follows.

- The first three time slots are allocated for transmitting messages to receiver 1 . At time slot $1, \mu_{1}^{[1]}, \nu_{1}^{[1]}$ and $\gamma_{1}^{[1]}$ 
are transmitted by transmitters 1,2 and 3 , respectively. At time slot 2 , transmitters 1 and 3 send new messages $\mu_{2}^{[1]}$ and $\gamma_{2}^{[1]}$, respectively. But transmitter 2 repeats its previous message $\nu_{1}^{[1]}$. At time slot 3, transmitters 1 and 2 send two new messages $\mu_{3}^{[1]}$ and $\nu_{2}^{[1]}$, respectively. Rotationally, transmitter 3 repeats its previous message $\gamma_{2}^{[1]}$ in this slot. Thus, 7 independent messages are sent in the first three time slots. The received equations at receiver $i$ are $L_{1}^{[i]}\left(\mu_{1}^{[1]}, \nu_{1}^{[1]}, \gamma_{1}^{[1]}\right)$, $L_{2}^{[i]}\left(\mu_{2}^{[1]}, \nu_{1}^{[1]}, \gamma_{2}^{[1]}\right), L_{3}^{[i]}\left(\mu_{3}^{[1]}, \nu_{2}^{[1]}, \gamma_{2}^{[1]}\right), i=1,2,3$. These linear equations are:

$$
\begin{aligned}
& L_{1}^{[i]}\left(\mu_{1}^{[1]}, \nu_{1}^{[1]}, \gamma_{1}^{[1]}\right)=h_{i 1}(1) \mu_{1}^{[1]}+h_{i 2}(1) \nu_{1}^{[1]}+h_{i 3}(1) \gamma_{1}^{[1]} \\
& L_{2}^{[i]}\left(\mu_{2}^{[1]}, \nu_{1}^{[1]}, \gamma_{2}^{[1]}\right)=h_{i 1}(2) \mu_{2}^{[1]}+h_{i 2}(2) \nu_{1}^{[1]}+h_{i 3}(2) \gamma_{2}^{[1]} \\
& L_{3}^{[i]}\left(\mu_{3}^{[1]}, \nu_{2}^{[1]}, \gamma_{2}^{[1]}\right)=h_{i 1}(3) \mu_{3}^{[1]}+h_{i 2}(3) \nu_{2}^{[1]}+h_{i 3}(3) \gamma_{2}^{[1]}
\end{aligned}
$$

- The next three time slots $(4,5,6)$ are allocated for transmitting messages to receiver $2 . \mu_{1}^{[2]}, \mu_{1}^{[2]}$ and $\mu_{2}^{[2]}$ are sent by transmitter 1 at time slots 4,5 and 6 , respectively. $\nu_{1}^{[2]}, \nu_{2}^{[2]}$ and $\nu_{3}^{[2]}$ are sent by transmitter 2 at each time slot, respectively. $\gamma_{1}^{[2]}, \gamma_{2}^{[2]}$ and $\gamma_{2}^{[2]}$ are sent by transmitter 3 at each time slot. Eventually, 7 independent messages are sent in the second round. After the second round the received equations at receiver $i$ can be represented as $L_{4}^{[i]}\left(\mu_{1}^{[2]}, \nu_{1}^{[2]}, \gamma_{1}^{[2]}\right)$, $L_{5}^{[i]}\left(\mu_{1}^{[2]}, \nu_{2}^{[2]}, \gamma_{2}^{[2]}\right), L_{6}^{[i]}\left(\mu_{2}^{[2]}, \nu_{3}^{[2]}, \gamma_{2}^{[2]}\right), i=1,2,3$. Similarly, we can represent these linear equations as follows:

$$
\begin{aligned}
& L_{4}^{[i]}\left(\mu_{1}^{[2]}, \nu_{1}^{[2]}, \gamma_{1}^{[2]}\right)=h_{i 1}(4) \mu_{1}^{[2]}+h_{i 2}(4) \nu_{1}^{[2]}+h_{i 3}(4) \gamma_{1}^{[2]} \\
& L_{5}^{[i]}\left(\mu_{1}^{[2]}, \nu_{2}^{[2]}, \gamma_{2}^{[2]}\right)=h_{i 1}(5) \mu_{1}^{[2]}+h_{i 2}(5) \nu_{2}^{[2]}+h_{i 3}(5) \gamma_{2}^{[2]} \\
& L_{6}^{[i]}\left(\mu_{2}^{[2]}, \nu_{3}^{[2]}, \gamma_{2}^{[2]}\right)=h_{i 1}(6) \mu_{2}^{[2]}+h_{i 2}(6) \nu_{3}^{[2]}+h_{i 3}(6) \gamma_{2}^{[2]}
\end{aligned}
$$

- The third three time slots $(7,8,9)$ are allocated for transmitting messages to receiver-3. $\mu_{1}^{[3]}, \mu_{2}^{[3]}$ and $\mu_{2}^{[3]}$ are sent by transmitter 1 at time slots 7,8 and 9 , respectively. $\nu_{1}^{[3]}, \nu_{1}^{[3]}$ and $\nu_{2}^{[3]}$ are sent by transmitter 2 at each time slot, respectively. $\gamma_{1}^{[3]}, \gamma_{2}^{[3]}$ and $\gamma_{3}^{[3]}$ are sent by transmitter 3 at each time slot. Hence, 7 independent messages are sent in the third round as well. The received equations at each receiver can be represented as $L_{7}^{[i]}\left(\mu_{1}^{[3]}, \nu_{1}^{[3]}, \gamma_{1}^{[3]}\right), L_{8}^{[i]}\left(\mu_{2}^{[3]}, \nu_{1}^{[3]}, \gamma_{2}^{[3]}\right)$, $L_{9}^{[i]}\left(\mu_{2}^{[3]}, \nu_{2}^{[3]}, \gamma_{3}^{[3]}\right), i=1,2,3$. These linear equations are:

$L_{7}^{[i]}\left(\mu_{1}^{[3]}, \nu_{1}^{[3]}, \gamma_{1}^{[3]}\right)=h_{i 1}(7) \mu_{1}^{[3]}+h_{i 2}(7) \nu_{1}^{[3]}+h_{i 3}(7) \gamma_{1}^{[3]}$
$L_{8}^{[i]}\left(\mu_{2}^{[3]}, \nu_{1}^{[3]}, \gamma_{2}^{[3]}\right)=h_{i 1}(8) \mu_{2}^{[3]}+h_{i 2}(8) \nu_{1}^{[3]}+h_{i 3}(8) \gamma_{2}^{[3]}$
$L_{9}^{[i]}\left(\mu_{2}^{[3]}, \nu_{2}^{[3]}, \gamma_{3}^{[3]}\right)=h_{i 1}(9) \mu_{2}^{[3]}+h_{i 2}(9) \nu_{2}^{[3]}+h_{i 3}(9) \gamma_{3}^{[3]}$

After phase one, we have the following observations:

- Message sharing: Suppose the backhaul can provide $M(3)$ to transmitter 1 so that after the first phase $\{\mu, \gamma\}$ appears at transmitter 1 , where $\{\mu, \gamma\}$ represents the message set $M(1)$ and $M(3)$. Similarly, $\{\mu, \nu\}$ appears at transmitter 2 , and $\{\nu, \gamma\}$ is available at transmitter 3 . Based on this observation, it is possible for the transmitters to recover the linear equations with corresponding known messages.

- Received linear equations: Clearly, every receiver has obtained 9 linear equations but only 3 of them are desired for decoding. That is, receiver 1 has $L_{1}^{[1]}, L_{2}^{[1]}$, and $L_{3}^{[1]}$; receiver 2 has $L_{4}^{[2]}, L_{5}^{[2]}$, and $L_{6}^{[2]}$; receiver 3 has $L_{7}^{[3]}, L_{8}^{[3]}, L_{9}^{[3]}$. In order to decode its desired 7 variables, 4 more independent equations are desired by each receiver. However, in order to utilize delayed CSIT, transmitters need to recover the proper overheard equations at receivers for efficiency. Yet, all the equations combine the messages from 3 different transmitters, while each transmitter is able to know the messages from 2 transmitters. For instance, equation $L_{1}^{[1]}$ can not be recovered by any transmitter, simply because it has 3 variables $\mu_{1}^{[1]}, \nu_{1}^{[1]}, \gamma_{1}^{[1]}$ whereas each transmitter just knows 2 of them. The problem is how to recover the desired equations at transmitters. Note that in the first phase, we repeated transmitting messages at time slot $2,3,5,6,8,9$. This gives us a hint for the following operations: combining $L_{1}^{[2]}$ and $L_{2}^{[2]}$, then by Gaussian eliminating $\nu_{1}^{[1]}$ a new linear equation $E_{1}^{[2]}\left(\mu_{1}^{[1]}, \mu_{2}^{[1]}, \gamma_{1}^{[1]}, \gamma_{2}^{[1]}\right)$ can be formed and it can be recover by transmitter 1 . We use $\left(L_{1}^{[2]}, L_{2}^{[2]}\right)$ to represent the linear equation after Gaussian elimination. Thus, $E_{1}^{[2]}\left(\mu_{1}^{[1]}, \mu_{2}^{[1]}, \gamma_{1}^{[1]}, \gamma_{2}^{[1]}\right)=\left(L_{1}^{[2]}, L_{2}^{[2]}\right)$. Similarly, we combine $L_{2}^{[2]}$ and $L_{3}^{[2]}$ together to get a new linear equation $E_{2}^{[2]}\left(\mu_{2}^{[1]}, \mu_{3}^{[1]}, \nu_{1}^{[1]}, \nu_{2}^{[1]}\right)$ by eliminating $\gamma_{2}^{[1]}$. $E_{2}^{[2]}\left(\mu_{2}^{[1]}, \mu_{3}^{[1]}, \nu_{1}^{[1]}, \nu_{2}^{[1]}\right)=\left(L_{2}^{[2]}, L_{3}^{[2]}\right) . E_{1}^{[2]}$ can be recovered by transmitter 2 . Repeatedly doing this across receivers, we define the following combinations:

$$
\begin{aligned}
& E_{3}^{[2]}\left(\mu_{1}^{[3]}, \mu_{2}^{[3]}, \gamma_{1}^{[3]}, \gamma_{2}^{[3]}\right)=\left(L_{7}^{[2]}, L_{8}^{[2]}\right) ; \\
& E_{4}^{[2]}\left(\nu_{1}^{[3]}, \nu_{2}^{[3]}, \gamma_{2}^{[3]}, \gamma_{3}^{[3]}\right)=\left(L_{8}^{[2]}, L_{9}^{[2]}\right) ; \\
& E_{1}^{[1]}\left(\nu_{1}^{[2]}, \nu_{2}^{[2]}, \gamma_{1}^{[2]}, \gamma_{2}^{[2]}\right)=\left(L_{4}^{[1]}, L_{5}^{[1]}\right) ; \\
& E_{2}^{[1]}\left(\mu_{1}^{[2]}, \mu_{2}^{[2]}, \nu_{2}^{[2]}, \nu_{3}^{[2]}\right)=\left(L_{5}^{[1]}, L_{6}^{[1]}\right) ; \\
& E_{3}^{[1]}\left(\mu_{1}^{[3]}, \mu_{2}^{[3]}, \gamma_{1}^{[3]}, \gamma_{2}^{[3]}\right)=\left(L_{7}^{[1]}, L_{8}^{[1]}\right) ; \\
& E_{4}^{[1]}\left(\nu_{1}^{[3]}, \nu_{2}^{[3]}, \gamma_{2}^{[3]}, \gamma_{3}^{[3]}\right)=\left(L_{8}^{[1]}, L_{9}^{[1]}\right) ; \\
& E_{1}^{[3]}\left(\mu_{1}^{[1]}, \mu_{2}^{[1]}, \gamma_{1}^{[1]}, \gamma_{2}^{[1]}\right)=\left(L_{1}^{[3]}, L_{2}^{[3]}\right) ; \\
& E_{2}^{[3]}\left(\mu_{2}^{[1]}, \nu_{3}^{[1]}, \nu_{1}^{[1]}, \nu_{2}^{[1]}\right)=\left(L_{2}^{[3]}, L_{3}^{[3]}\right) ; \\
& E_{3}^{[3]}\left(\nu_{1}^{[2]}, \nu_{2}^{[2]}, \gamma_{1}^{[2]}, \gamma_{2}^{[2]}\right)=\left(L_{4}^{[2]}, L_{5}^{[2]}\right) ; \\
& E_{4}^{[3]}\left(\mu_{1}^{[2]}, \mu_{2}^{[2]}, \nu_{2}^{[2]}, \nu_{3}^{[2]}\right)=\left(L_{5}^{[2]}, L_{6}^{[2]}\right) .
\end{aligned}
$$

Because of message sharing, transmitter 1 can recover $E_{1}^{[2]}$, $E_{1}^{[3]}, E_{3}^{[1]}$ and $E_{3}^{[2]}$; transmitter 2 can recover $E_{2}^{[1]}, E_{2}^{[2]}, E_{2}^{[3]}$ and $E_{4}^{[3]}$; and transmitter 3 can recover $E_{1}^{[1]}, E_{4}^{[1]}, E_{4}^{[2]}$ and $E_{3}^{[3]}$.

- Desired equations: If receiver 1 can obtain the combinations $E_{1}^{[2]}, E_{2}^{[2]}, E_{1}^{[3]}$ and $E_{2}^{[3]}$, then its desired 7 variables can be decoded almost surely collaborating with 3 received equations. Similarly, receiver 2 desires $E_{1}^{[1]}, E_{2}^{[1]}, E_{3}^{[3]}$ and $E_{4}^{[3]}$; receiver 3 desires $E_{3}^{[2]}, E_{4}^{[2]}, E_{3}^{[1]}$, and $E_{4}^{[1]}$. The purpose of the second phase is to deliver these equations to their desired receivers. For specific use of these above combinations, we introduce Phase Two in what follows.

Phase Two: 6 time slots are allocated in this phase. At first, we define 6 order-two messages as $m_{12}(1)=$ $\left(E_{1}^{[1]}, E_{1}^{[2]}\right), m_{12}(2)=\left(E_{2}^{[1]}, E_{2}^{[2]}\right), m_{13}(1)=\left(E_{1}^{[3]}, E_{3}^{[1]}\right)$, $m_{13}(2)=\left(E_{2}^{[3]}, E_{4}^{[1]}\right), m_{23}(1)=\left(E_{3}^{[2]}, E_{3}^{[3]}\right)$, and $m_{23}(2)=$ $\left(E_{4}^{[2]}, E_{4}^{[3]}\right)$. In the above representations, $m_{i k}$ means the 


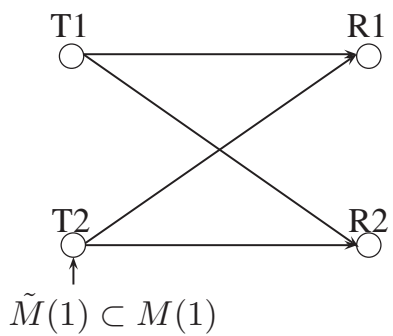

Fig. 3. Two user neighbor-node partially cooperative $\mathrm{X}$ channel with delayed CSIT (feedback links are omitted for concision).

order-two message intending for receiver $i$ and $k$ and the right hand side means a linear combination of the two terms in the brackets. The principle of this phase is to deliver 1 ordertwo message at each time slot. Specifically, at time slot 10 , by transmitter 1 sending $E_{1}^{[2]}$, transmitter 3 sending $E_{1}^{[1]}$ and transmitter 2 remaining silence, the received signal of receiver $i$ is $h_{i 1}(10) E_{1}^{[2]}+h_{i 3}(10) E_{1}^{[1]}, i=1,2$. Thus, $m_{12}(1)$ is delivered. Since receiver 1 knows $E_{1}^{[1]}$, it can extract it from $m_{12}(1)$ to obtain its desired $E_{1}^{[2]}$. Meanwhile, receiver 2 can eliminate $E_{1}^{[2]}$ to obtain its desired $E_{1}^{[1]}$. Similarly, $m_{12}(2)$, $m_{13}(1), m_{13}(2), m_{23}(1), m_{23}(2)$ are sent at time slots 11 , $12,13,14,15$, respectively. We omit the transmission details here.

After these 6 time slots in phase two, we observe that every receiver has collected 4 order-two messages, i.e., receiver 1 has collected $m_{12}(1), m_{12}(2), m_{13}(1)$ and $m_{13}(2)$; receiver 2 has collected $m_{12}(1), m_{12}(2), m_{23}(1)$ and $m_{23}(2)$; receiver 3 has collected $m_{13}(1), m_{13}(2), m_{23}(1)$ and $m_{23}(2)$. By eliminating the overheard equations from the order-two messages, every receiver is able to obtain 4 independent equations. Collaborating with the 3 equations received in phase one, 7 variables can be decoded by each receiver almost surely. As conclusion, 21 independent messages are transmitted in 15 time slots. DoF $\frac{7}{5}$ can be achieved.

The generalization from 3 users to more users is difficult and we leave it for future work. Clearly, Theorem 2 is obtained by assuming $\tilde{M}(i)=M(i)$, for $i=1,2,3$. In the next subsection, we start to consider the DoF gain when $\tilde{M}(i) \subset M(i)$.

\section{B. One Example: Subset Message Sharing Can Provide DoF Gain}

A 2 user neighbor cooperative $\mathrm{X}$ network is illustrated in Fig. 3, where message set $\tilde{M}(1)$ is available at transmitter 2 . Clearly, when $\tilde{M}(1)=M(1)$, the optimal DoF $\frac{4}{3}$ is available by the scheme in [5]. In what follows, we show that even when a proper subset $\tilde{M}(1) \subset M(1)$ appears at transmitter 2 , DoF improvement can be obtained with delayed CSIT. We give one example to interpret the scheme in which DoF $\frac{5}{4}$ can be achieved which outperforms $\frac{6}{5}$ in [6] without cooperation.

Transmission Design: A two-phase transmission is designed in this scenario.

Phase One: The first phase needs three time slots. For the first time slot, transmitter 1 sends $\mu_{1}$ and transmitter 2 sends $\nu_{1}$ both intending for receiver 2 . The received equation at receiver 1 is $L_{1}^{[1]}\left(\mu_{1}, \nu_{1}\right)$ while the received equation at receiver two is $L_{1}^{[2]}\left(\mu_{1}, \nu_{1}\right)$. At the second time slot, $\mu_{2}$ and $\nu_{2}$ are sent by transmitter 1 and 2 , respectively, both intending for receiver 1 . $L_{2}^{[1]}\left(\mu_{2}, \nu_{2}\right)$ and $L_{2}^{[2]}\left(\mu_{2}, \nu_{2}\right)$ are received by receiver 1 and 2 at the second time slot. Now we suppose $\mu_{1}$ can be obtained by transmitter 2, and the next time slot is designed as follows. A new message $\mu_{3}$ is sent by transmitter 1 intending for receiver 1 while transmitter 2 repeats $\nu_{2} . L_{3}^{[1]}\left(\mu_{3}, \nu_{2}\right)$ and $L_{3}^{[2]}\left(\mu_{3}, \nu_{2}\right)$ appear at receiver 1 and 2, respectively. We combine $L_{2}^{[2]}$ and $L_{3}^{[2]}$ together to obtain a new linear equation $E\left(\mu_{2}, \mu_{3}\right)$ by eliminating $\nu_{2}$, and $E\left(\mu_{2}, \mu_{3}\right)$ can be recovered by receiver 1 at the next time slot.

Phase Two: The second phase needs one time slot. At time slot $4, E\left(\mu_{2}, \mu_{3}\right)$ is sent at transmitter 1 and $L_{1}^{[1]}\left(\mu_{1}, \nu_{1}\right)$ is sent at transmitter 2 . As results, the linear combination $\left(E\left(\mu_{2}, \mu_{3}\right), L_{1}^{[1]}\left(\mu_{1}, \nu_{1}\right)\right)$ appears at each receiver. For receiver 1 , by eliminating $L_{1}^{[1]}, \mu_{2}, \mu_{3}$ and $\nu_{2}$ can be decoded by solving equation group $E\left(\mu_{2}, \mu_{3}\right), L_{2}^{[1]}\left(\mu_{2}, \nu_{2}\right)$ and $L_{3}^{[1]}\left(\mu_{3}, \nu_{2}\right)$. On the other hand, for receiver $2, \mu_{1}, \nu_{1}$ can be resolved by eliminating $E\left(\mu_{2}, \mu_{3}\right)$ to obtain $L_{1}^{[1]}\left(\mu_{1}, \nu_{1}\right)$ and collaborating with $L_{1}^{[2]}\left(\mu_{1}, \nu_{1}\right)$. Thus DoF $\frac{5}{4}$ is achieved almost surely.

\section{Conclusions}

We studied the achievable DoF of partially cooperative $K$-user X networks with delayed CSIT. Compared to noncooperative scenarios, the DoF gain can be obtained if one super-node exists in the network. If the super-node is absent, we propose a neighbor node partial cooperation scheme to achieve higher DoF for a small number of $K$, i.e., $K=3$. Finally, we show that subset message sharing is still useful for DoF improvement in $\mathrm{X}$ networks. Our results show the benefits of source cooperation even when only a part of messages can be shared among transmitters.

\section{REFERENCES}

[1] V. R. Cadame and S. A. Jafar, 'Interference alignmen and the degrees of freedom of wireless X networks," IEEE Trans. Inf. Theory, vol. 55, no. 9, pp. 3893-3908, Sep. 2009

[2] A. Goldsmith, S. A. Jafar, N. Jindal and S. Vishwanath, "Capacity limits of MIMO Channels," IEEE Jour. Seleted Areas in Coтmu., vol. 21, no. 5, pp. 684-702, Jun. 2003.

[3] N. Jindal, "MIMO broadcast channels with finite-rate feedback," IEEE Trans. Inf. Theory, vol. 52, no. 11, pp. 5045-5060, Nov, 2006.

[4] J. Thukral and H. Bolcskei, "Interference alignment with limited feedback," IEEE Intern. Symp. Inform. Theory, Seoul, Korea, Jun, 2009.

[5] M. A. Maddah-Ali and D. Tse, "Completely stale transmitter channel state information is still very useful," Forty-Eighth Annual Allerton Conference on Communication, Control, and Computing, Allerton Retreat Center, Monticello, Illinois, Sept. 2010.

[6] A. Ghasemi, A. S. Motahari and A. K. Khandani, "On the degrees of freedom of X channel with delayed CSIT," IEEE Intern. Symp. Inform. Theory, St. Peterburg, Russia, Aug. 2011.

[7] M. J. Abdoli, A. Ghasemi and A. K. Khandani, "On the Degrees of Freedom of K-User SISO Interference and X Channels with Delayed CSIT," available online at http://arxiv.org/pdf/1109.4314, Sep, 2011.

[8] S. A. Jafar and S. Shamai, "Degrees of freedom region of the MIMO X channel," IEEE Trans. Inf. Theory, vol. 54, no. 1, pp. 151-170, Jan, 2008. 